\title{
Estimação de Fatores de Correção do Perímetro Escrotal para Idade e Peso Corporal em Touros Jovens da raça Nelore ${ }^{1}$
}

\author{
Carlos Darío Ortiz Peña², Sandra Aidar de Queiroz ${ }^{3}$, Luiz Alberto Fries ${ }^{4,5}$
}

\begin{abstract}
RESUMO - Neste trabalho foram estudados os efeitos de idade e peso sobre o perímetro escrotal (PE) de touros jovens da raça Nelore e estimados os fatores de correção (FC) do PE para esses efeitos. As informações utilizadas pertencem aos Registros de Produção da Associação Paraguaia dos Criadores de Nelore, obtidos entre 1986 e 1997, de 110 criatórios, totalizando 7458 observações. Os coeficientes de regressão que permitiram o cálculo dos FCs para o PE foram estimados pelo método dos quadrados mínimos, empregando-se um modelo que considerou o grupo contemporâneo (animais nascidos no mesmo ano, estação, rebanho, avaliados na mesma data e do mesmo grupo de manejo alimentar na desmama e no sobreano), o efeito linear da idade em dias e os efeitos linear e quadrático do peso aos 570 dias de idade (P570). O efeito da idade sobre o PE foi igual a 0,0383( $\pm 0,0024) \mathrm{cm} /$ dia e do P570 sobre o PE corrigido para essa idade, $0,0708( \pm 0,0049) \mathrm{cm} / \mathrm{kg}$, efeito linear, e $-0,0000413( \pm 0,0000074) \mathrm{cm} / \mathrm{kg}^{2}$, efeito quadrático. A idade e o peso do animal foram importantes fontes de variação a serem removidas do PE, como indicativo da precocidade sexual de touros de sobreano.
\end{abstract}

Palavras-chave: coeficientes de regressão, desempenho reprodutivo, gado de corte, precocidade sexual, puberdade

\section{Estimation of Correction Factors of Scrotal Circumference for Age and Body Weight in Young Nellore Breed Bulls}

\begin{abstract}
This work studied the effects of age and weight on scrotal circumference (SC) of Nellore young breed bulls, and also estimated the SC correction factors (CF) for those effects. The information came from the Production Registry of the Paraguayan Nellore Breeders Association, obtained from 1986 to 1997 from 110 herds, totalling 7,458 observations. The regression coefficients that allowed the calculation of CF for SC were estimated by the least square methods. The model considered the contemporary group (animals born in the same year, season, herd, evaluated in the same day and from the same weaning and yearling feed management group), linear effect of age of the animal (days), and linear and quadratic effects of live weight at 570 days of age (W570). The age effect on SC was $0.0383( \pm 0.0024) \mathrm{cm} / \mathrm{day}$, and of W570 on SC, corrected for this age, was $0.0708( \pm 0.0049) \mathrm{cm} / \mathrm{kg}$, linear effect, and -0.0000413 $( \pm 0.0000074) \mathrm{cm} / \mathrm{kg}^{2}$, quadratic effect. The age and live weight of the animals were important sources of variation to be removed from the $\mathrm{SC}$ as an indicator of sexual precocity in yearling bulls.
\end{abstract}

Key Words: beef cattle, puberty, regression coefficients, reproductive performance, sexual precocity

\section{Introdução}

O desempenho reprodutivo dos animais é um dos principais fatores determinantes da eficiência total de produção da bovinocultura de corte e, portanto, deve ser considerado nos programas de melhoramento genético (ALENCAR et al., 1993).

Entretanto, algumas práticas de manejo reprodutivo rotineiramente empregadas na pecuária de corte, como a estação de monta, dificultam a estimação mais exata da herdabilidade de algumas características de fertilidade das fêmeas que seriam facilmente mensuráveis a campo, como a idade ao primeiro parto
(ALBUQUERQUE e FRIES, 1998) e o intervalo de parto (BARROS et al., 1996). Este fato, aliado à baixa intensidade de seleção normalmente aplicada às fêmeas, resulta em pequeno progresso genético esperado para as características reprodutivas (ALENCAR et al., 1993). Nesse contexto, estudos relacionados à função reprodutiva dos machos devem contribuir para o estabelecimento de programas de manejo e de seleção mais adequados ao aprimoramento da pecuária, principalmente, nos sistemas de produção em que a reprodução é feita por monta natural, pois, nesses casos, a fertilidade dos touros é de especial importância na determinação da eficiên-

\footnotetext{
1 Parte da dissertação de mestrado apresentada pelo primeiro autor, ao curso de PG-Zootecnia/Genética e Melhoramento Animal - FCAV/UNESP.

2 Programa de estudante convênio (PEC-PG/96) Brasil - Paraguai/CAPES. E.mail: cadop@rieder.net.py

3 Depto. Zootecnia, FCAV/UNESP - Jaboticabal. E-mail. saquei@fcav.unesp.br - Bolsista do CNPq.

4 Gensys Consultores Associados S/C Ltda. E.mail: fries@fcav.unesp.br

5 Professor visitante - CAPES - DZ - FCAV - UNESP.
} 
cia de produção (PINEDA et al., 1998).

A medida perimetral da maior parte transversal da bolsa escrotal é considerada uma característica contínua, denominada Perímetro Escrotal (PE). Este PE é, em animais jovens, indicador útil do tamanho testicular, da capacidade de produção espermática, das características físicas do sêmen, da idade à puberdade e, também, da fertilidade dos machos, bem como das fêmeas aparentadas, principalmente meioirmãs e filhas (BRINKS et al., 1978; COULTER e KELLER, 1982; TOELLE e ROBINSON, 1985; ALENCAR et al., 1993; ARIAS e SLOBODZIAN, 1998; e GRESSLER et al., 1998).

Procurando marcadores de precocidade em gado Nelore, UNANIAM (1997) utilizou, como conceito de desenvolvimento reprodutivo dos machos, a idade em que aparecem os primeiros espermatozóides no ejaculado. Assim, o autor constatou, nesta raça, o aparecimento dos primeiros espermatozóides vivos a partir dos 10,6 meses de idade, variando até os 16 meses, sendo a média igual a 13,6 meses de idade com 22,9 cm de PE. Segundo SILVA et al. (1993), o crescimento mais intenso dos testículos ocorre próximo à puberdade, indicando que a tomada da medida do PE neste período é estratégica para avanços genéticos em fertilidade e precocidade sexual (LÔBO et al., 1994; DAL-FARRA et al., 1998).

Analisando grande número de informações de touros Nelore, ARIAS e SLOBODZIAN (1998) notaram que a melhoria no manejo produziu incremento tanto no PE como no peso corporal (PC). Verificaram, entretanto, que o PC evoluiu mais rapidamente que o PE, quando os níveis de alimentação foram melhorados, e que, à mesma idade e potencial de desenvolvimento sexual, animais melhor alimentados apresentaram relação mais favorável para o ganho de peso do que para o PE. Observaram, também, que o efeito da idade dos touros Nelore foi fonte importante de variação e cada dia de idade acima da média (570 dias) ocasionou aumento de $0,0068 \mathrm{~cm}$ no PE. Relataram ainda que, para cada $\mathrm{kg}$ de desvio da média de peso aos 570 dias de idade, ocorreu aumento de 0,0394 cm no PE. Os autores concluíram que diferenças no desenvolvimento testicular aos 570 dias de idade estariam relacionadas com diferenças em precocidade, sobretudo se maiores perímetros testiculares corresponderem a animais de mesmo tamanho corporal. Assim, para as mesmas condições corporal e de peso, o desejável seriam relações de PC/PE menores, visando identificar os animais sexualmente precoces.
Outros parâmetros importantes a considerar são as correlações genéticas entre as características reprodutivas e as de desenvolvimento ponderal. MARTINS FILHO et al. (1994) e ELER et al.(1996) estimaram correlação genética baixa e positiva $(0,18)$ entre o PE e o peso à desmama (PD) de bovinos Nelore. ALENCAR e VIEIRA (1989), LÔBO et al. (1994) e QUIRINO e BERGMANN (1997) reportaram valores moderados para a correlação genética $(0,51$; 0,44 ; e 0,64 ; respectivamente) entre PE e PC ao sobreano, sugerindo a possibilidade de seleção simultânea destas características nos programas de melhoramento. Entretanto, segundo DAL-FARRA et al. (1998) e BRITO (1997), devido à estreita relação do PE com o desenvolvimento ponderal e a idade à puberdade, se a meta for obter progresso genético em precocidade sexual, seria recomendável a correção do PE para os efeitos de idade e PC do animal, a fim de evitar resposta correlacionada com maior tamanho adulto do animal.

Os objetivos deste trabalho foram estudar os efeitos da idade e do peso corporal do animal sobre o PE observado ao sobreano, em bovinos de raça Nelore no Paraguai, bem como estimar fatores de correção para o PE, em função dessas variáveis.

\section{Material e Métodos}

Trabalhou-se com informações de 110 rebanhos, obtidas entre os anos de 1986 e 1997, fornecidas pela Associação Paraguaia dos Criadores de Nelore. O PE foi mensurado na pesagem ao sobreano.

O grupo contemporâneo (GC) foi composto por touros jovens nascidos na mesma cabanha (proprietário), fazenda (rebanho), ano e estação, pesados e medidos, no mesmo dia, tanto à desmama quanto ao sobreano, e pertencentes ao mesmo grupo de manejo (GM) de desmama e sobreano. A data da avaliação foi incluída no GC visando agrupar os animais de manejo homogêneo, provenientes dos mesmos pastos, e que tenham recebido as mesmas influências de tratamento nos currais. As estações de nascimento consideradas foram 1 - janeiro a março, 2 - abril a junho, 3 - julho a setembro e 4 - outubro a dezembro. Os GMs corresponderam à informação do regime alimentar dada pelos níveis: 1 - pastagens nativas, 2 - pastagens nativas e cultivadas, 3- somente pastagens cultivadas, 4 - pastagens cultivadas e com suplementação (no inverno/temporária) e 5confinamento.

Foram descartados os registros de peso ao 
Rev. bras. zootec.

sobreano inferiores a $100 \mathrm{~kg}$ e todos os registros com observações anormais do PE. Foram, também, eliminados registros pertencentes a GCs e touros com menos que quatro observações.

Para a verificação da conexão entre os GCs, utilizou-se o programa MILC (Matriz de Incidência, Laços Genéticos e Conectabilidade) elaborado por FRIES e ROSO (1997), restando 7458 registros disponíveis para análise.

A correção do PE para idade (570 dias) e peso ao sobreano exigiu a padronização do peso ao sobreano para 570 dias de idade (P570), sendo esta última feita por meio da fórmula:

$$
\mathrm{P} 570=\mathrm{P} 205+\text { GDS }
$$

em que: P205 é peso padronizado para 205 dias de idade e GDS, ganho de peso dos 205 aos 570 dias de idade.

O P205 e o GDS foram calculados pelas equações:

$$
\begin{gathered}
\mathrm{P} 205=\mathrm{PN}+(\mathrm{GMD} \times 205) \\
\text { GDS }=\text { GMP } \times 365
\end{gathered}
$$

em que: PN é peso ao nascimento do animal; GMD, ganho médio diário na pré-desmama (corrigido para 205 dias de idade do bezerro, data juliana de nascimento e idade da mãe ao parto); e GMP, ganho médio diário observado no pós-desmame.

Os coeficientes de regressão para os efeitos de idade do animal e P570 foram estimados em dois processos, usando-se o procedimento GLM do SAS (SAS, 1996), por meio dos seguintes modelos estatísticos de efeitos fixos:

$$
\text { 1ㅇ) } \mathrm{y}_{i j}=\beta_{0}+\mathrm{GC}_{\mathrm{i}}+\beta_{1}\left(\mathrm{I}_{\mathrm{ij}}\right)+\mathrm{e}_{\mathrm{ij}}
$$

em que: $\mathrm{y}_{i j}$ é observação de PE do j-ésimo animal do i-ésimo grupo contemporâneo; $\beta_{0}$, intercepto; $\mathrm{GC}_{i}$, efeito do i-ésimo grupo contemporâneo; $\beta_{1}$, coeficiente de regressão linear; $\mathrm{I}_{i j}$, efeito da idade do j-ésimo animal pertencente ao i-ésimo GC; e e ${ }_{i j}$, erro aleatório associado àij-ésima observação, pressuposto normal e independentemente distribuído.

$$
\left.2^{2}\right) \mathrm{y}_{i j}^{\prime}=\beta_{0}+\mathrm{GC}_{\mathrm{i}}+\beta_{1}\left(\mathrm{P}_{\mathrm{ij}}\right)+\mathrm{b}_{2}(\mathrm{Pij})^{2}+\mathrm{e}_{\mathrm{ij}}
$$

em que: $y_{i j}^{\prime}$ é observação de PE corrigido para idade do j-ésimo animal (PEi) do i-ésimo grupo contemporâneo; $\beta_{0}$, intercepto; $\mathrm{GC}_{i}$, efeito do i-ésimo grupo contemporâneo; $\beta_{1}$ e $\beta_{2}$, coeficientes de regressão dos termos linear e quadrático do $\mathrm{PE}$, em função do peso aos 570 dias de idade, respectivamente; $\mathrm{P}_{i j}$, efeito do P570 do j-ésimo animal pertencente ao i-ésimo GC; e e ${ }_{i j}$, erro aleatório associado à ijésima observação, pressuposto normal e independentemente distribuído.

A seguir, o perímetro escrotal e o perímetro escrotal corrigido para 570 dias de idade foram preditos de acordo com as seguintes equações:

$$
\hat{P E} i=\mu_{\mathrm{PE}}+\beta_{1}\left(\mathrm{I}-\mu_{\mathrm{i}}\right)
$$

em que: $\hat{P E} i$, perímetro escrotal predito em função da idade do animal, em cm; $\mu_{P E}$, média geral observada do PE, em cm; $\hat{\beta}_{i}$, coeficiente de regressão linear estimado para a idade do animal; $I$, idade do animal no momento da mensuração do perímetro escrotal, em dias; $\mu_{i}$, média geral observada da idade do animal, em dias.

$$
P \hat{E i p}=\mu_{P E i}+\hat{\beta}_{1}\left(P 570-\mu_{P 570}\right)+\hat{\beta}_{2}\left(P 570-\mu_{P 570}\right)^{2}
$$

em que: PEip é perímetro escrotal corrigido para 570 dias de idade predito em função do peso corporal padronizado aos 570 dias de idade (P570), em cm; $\mu_{P E i}$, média do perímetro escrotal, em $\mathrm{cm}$, corrigido para 570 dias de idade (PEi); $\hat{\beta}$ e $\hat{\beta}_{2}$, coeficientes de regressão, linear e quadrático do $\mathrm{PEi}$, em função do P570, respectivamente; P570, peso padronizado do animal aos 570 dias de idade em kg; e $\mu_{\mathrm{P} 570}$, média geral observada do P570, em kg.

Analisando-se as distribuições das freqüências por idade e peso considerados, os fatores de correção foram estimados para intervalos de 530 a 610 dias de idade e para 160 a $590 \mathrm{~kg}$ de P570. Todos os animais acima ou abaixo destas classes extremas foram considerados como pertencentes a elas. Os fatores de correção para 570 dias de idade foram estimados por:

$$
\mathrm{FC}_{i}=\frac{P \hat{E i_{570}}}{\hat{P E i}}
$$

em que: $\mathrm{FC}_{i}$ é fator de correção para idade aos 570 dias; $P \hat{E i}_{570}$, valor predito do perímetro escrotal, em cm, para 570 dias de idade; e $\hat{P E i}$, valor predito do perímetro escrotal, em $\mathrm{cm}$, para a idade do animal.

Para P570, o fator de correção foi obtido pela fórmula:

$$
\text { FCip }=\frac{{\hat{P E i p_{310}}}}{P \hat{E i p}}
$$

em que: FCip é fator de correção para idade aos 570 dias e $310 \mathrm{~kg}$ de peso corporal (PC); PEip $_{310}$, valor predito para 570 dias de idade e $310 \mathrm{~kg}$ de PC; e PEip, valor predito para 570 dias de idade e o PC do animal em questão. 


\section{Resultados e Discussão}

Na Tabela 1, encontram-se as estatísticas descritivas das características perímetro escrotal, idade e peso dos animais ao sobreano. Os valores observados foram semelhantes aos relatados por MARTINS FILHO et al. (1994), LÔBO et al. (1994), ELER et al.(1996), QUIRINO e BERGMANN (1997) e DAL FARRA et al. (1998) para a raça Nelore.

Nas Figuras 1 e 2, encontram-se apresentadas as médias das observações do PE, em função da idade e do P570, respectivamente. Pode-se verificar que, em um intervalo de 80 dias, as médias de PE aumentaram linearmente, aproximadamente, $2 \mathrm{~cm}$ (Figura 1) enquanto, para um intervalo de $450 \mathrm{~kg}$ de P570, praticamente dobraram de tamanho (Figura 2).

Na Tabela 2, encontram-se os resultados prelimi- nares da análise de variância do PE, considerando-se os efeitos de idade e do P570. Constata-se que, entre os efeitos considerados no modelo de análise do PE, o único que resultou ser não significativo $(P>0,05)$ foi a idade do animal, como efeito quadrático. Assim, o PE foi corrigido para os efeitos linear da idade e linear e quadrático do peso corporal. DAL-FARRA et al. (1998), em touros Nelore, e BRITO (1997), com animais Polled Hereford, também verificaram efeitos significativos linear e linear e quadrático, respectivamente, da idade e do peso do animal sobre o PE.

Na Tabela 3, encontra-se um resumo das análises de variância realizadas com o intuito de verificar qual seria o modelo mais adequado para a estimação dos coeficientes de regressão utilizados nos cálculos dos FCs do PE. Observa-se que a inclusão do GC, em adição à idade do animal no modelo de análise do PE,

Tabela 1 -Estatísticas descritivas das características perímetro escrotal, peso e idade ao sobreano de touros jovens da raça Nelore, no Paraguai

Table 1 - Descriptive statistic of the traits scrotal circumference, weight and age at yearling of young Nellore bulls, in Paraguay

\begin{tabular}{lcccccc}
\hline $\begin{array}{l}\text { Característica } \\
\text { Trait }\end{array}$ & $\mathrm{N}$ & $\begin{array}{c}\text { Média } \\
\text { Average }\end{array}$ & $\begin{array}{c}\mathrm{DP}^{1} \\
\mathrm{SD}^{1}\end{array}$ & $\mathrm{CV}^{2}(\%)$ & $\begin{array}{c}\text { Mínimo } \\
\text { Minimum }\end{array}$ & $\begin{array}{c}\text { Máximo } \\
\text { Maximum }\end{array}$ \\
\hline $\begin{array}{l}\text { Idade }(\text { dias }) \\
\text { Age }(\text { days })\end{array}$ & 7458 & 574 & 19 & 3,28 & 519 & 663 \\
$\begin{array}{l}\mathrm{P} 570^{3}(\mathrm{~kg}) \\
W 570^{3}(\mathrm{~kg})\end{array}$ & 7458 & 310 & 57 & 18,30 & 154 & 594 \\
$\mathrm{PE}^{4}(\mathrm{~cm})$ & 7458 & 25,71 & 3,54 & 13,76 & 13 & 40 \\
$S C^{4}(\mathrm{~cm})$ & & & & & & \\
\hline
\end{tabular}

${ }_{1}^{1}$ Desvio-padrão; ${ }^{2}$ coeficiente de variação; ${ }^{3}$ peso padronizado aos 570 dias de idade; ${ }^{4}$ perímetro escrotal observado.

1 Standard deviation; ${ }^{2}$ coefficient of variation; ${ }^{3}$ weight at 570 days of age; ${ }^{4}$ observed scrotal circumference.

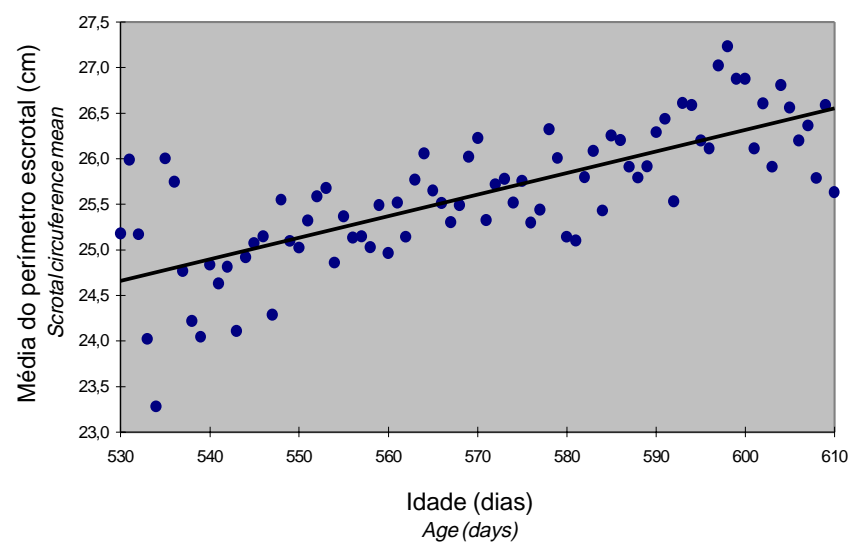

Figura 1 - Média do perímetro escrotal observado em função da idade de touros jovens Nelore, no Paraguai.

Figure 1 - Observed scrotal circumference mean, according to the age of young Nellore bulls, in Paraguay.

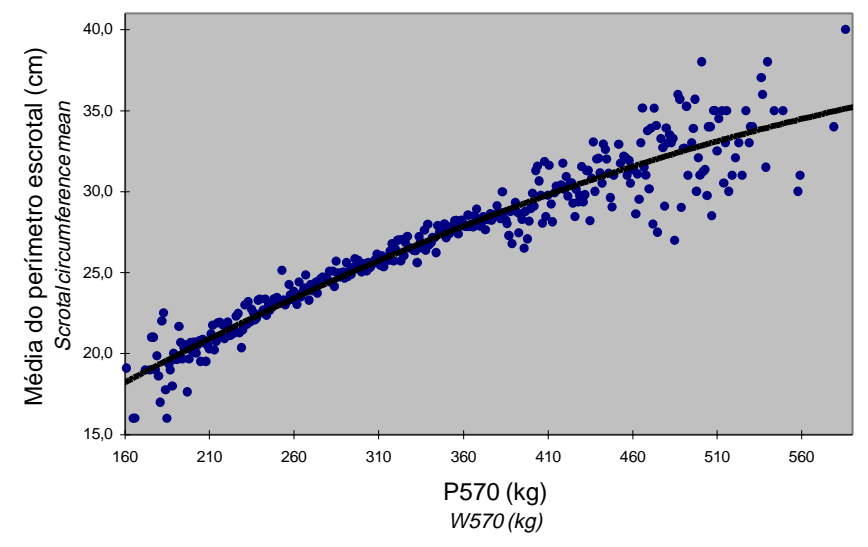

Figura 2 - Média do perímetro escrotal observado, em função do peso corporal padronizado para 570 dias de idade (P570) de touros jovens Nelore, no Paraguai.

Figure 2 - Observed scrotal circumference mean, according to the body weight at 570 days of age (W570) of young Nellore bulls, in Paraguay. 
Rev. bras. zootec.

Tabela 2 - Resumo da análise de variância do perímetro escrotal, pelo método dos quadrados mínimos, em touros jovens Nelore, no Paraguai

Table 2 - Summary of analysis of variance for scrotal circumference (SC), by least squares method, in young Nellore bulls, in Paraguay

\begin{tabular}{|c|c|c|}
\hline $\begin{array}{l}\text { Fatores de variação } \\
\text { Source of variation }\end{array}$ & $\begin{array}{l}\text { Graus de liberdade } \\
\text { Degree of freedom }\end{array}$ & $\begin{array}{l}\text { Quadrados médios } \\
\text { Mean squares }\end{array}$ \\
\hline Modelo & 405 & $145,799 *$ \\
\hline Model & & \\
\hline Grupo contemporâneo & 401 & $110,059 *$ \\
\hline Contemporary group & & \\
\hline $\begin{array}{l}\text { Idade do animal (linear) } \\
\text { Age of animal (linear) }\end{array}$ & 1 & $1.723,287 *$ \\
\hline $\begin{array}{l}\text { Idade do animal (quadrático) } \\
\text { Age of animal (quadratic) }\end{array}$ & 1 & 11,865 \\
\hline $\begin{array}{l}\mathrm{P}^{570^{1}} \text { (linear) } \\
W 570^{1} \text { (linear) }\end{array}$ & 1 & $13.023,339 *$ \\
\hline $\begin{array}{l}\text { P570 (quadrático) } \\
\text { W570 (quadratic) }\end{array}$ & 1 & $156,456^{*}$ \\
\hline $\begin{array}{l}\text { Resíduo } \\
\text { Error }\end{array}$ & 7052 & 4,871 \\
\hline $\mathrm{R}^{2}=0,63 ; \mathrm{CV}=8,58 \% ; \sigma=2$ & $=25,71 \mathrm{~cm}$ & \\
\hline
\end{tabular}

Tabela 3 - Coeficientes de regressão estimados para a correção do perímetro escrotal (PE), para idade e peso corporal aos 570 dias (P570), pelo método dos quadrados mínimos e pelos procedimentos REG (modelo 1 ) e GLM (modelo 2), em touros jovens Nelore, no Paraguai

Table 3 - Estimated regression coefficients for scrotal circumference (SC), correction for age (1st step) and body weight (2 ${ }^{\text {nd }}$ step) at 570 days (W570), by least squares method and REG (model 1) using GLM (model 2) procedures, in young Nellore bulls, in Paraguay

\begin{tabular}{|c|c|c|c|c|}
\hline \multirow[b]{2}{*}{$\begin{array}{l}\text { Parâmetro } \\
\text { Parameter }\end{array}$} & \multicolumn{2}{|c|}{$\begin{array}{c}1^{\mathrm{a}} \text { etapa (idade) } \\
1^{\text {st }} \text { step (age) } \\
\end{array}$} & \multicolumn{2}{|c|}{$\begin{array}{l}2^{\mathrm{a}} \text { etapa }(\mathrm{P} 570) \\
2^{\text {nd }} \text { step }(\text { W570) } \\
\end{array}$} \\
\hline & $\begin{array}{c}\text { Mod.1 (s/GC } 1) \\
\text { Mod. } 1\left(\text { without } C G^{1}\right)\end{array}$ & $\begin{array}{c}\text { Mod.2(c/GC) } \\
\text { Mod.1 } 1 \text { with CG) }\end{array}$ & $\begin{array}{c}\text { Mod.1(s/GC) } \\
\text { Mod. } 1 \text { (without CG) }\end{array}$ & $\begin{array}{c}\text { Mod.2(c/GC) } \\
\text { Mod. } 1 \text { (with CG) }\end{array}$ \\
\hline $\begin{array}{l}\mathrm{b}_{0}(\text { intercepto }) \\
\mathrm{b}_{0}(\text { intercept })\end{array}$ & $11,05^{*}$ & $1,36^{*}$ & $8,12 *$ & $6,88^{*}$ \\
\hline $\begin{array}{l} \pm \text { e.p. } \\
\pm \text { s.e. }\end{array}$ & $\pm 1,26$ & $\pm 1,73$ & $\pm 0,61$ & $\pm 1,20$ \\
\hline $\begin{array}{l}\mathrm{b}_{1}(\text { linear }) \\
\mathrm{b}_{1}(\text { linear })\end{array}$ & $0,0255^{*}$ & $0,0383 *$ & $0,0695^{*}$ & $0,0708 *$ \\
\hline $\begin{array}{l} \pm \text { e.p. } \\
\pm \text { s.e. }\end{array}$ & $\pm 0,0022$ & $\pm 0,0024$ & $\pm 0,0037$ & $\pm 0,0049$ \\
\hline $\begin{array}{l}\mathrm{b}_{2} \text { (quadrático) } \\
\mathrm{b}_{2} \text { (quadratic) }\end{array}$ & - & - & $-0,0000415^{*}$ & $-0,0000413^{*}$ \\
\hline $\begin{array}{l} \pm \text { e.p. } \\
\pm \text { s.e. }\end{array}$ & - & - & $\pm 0,0000055$ & $\pm 0,0000074$ \\
\hline $\begin{array}{l}\text { Soma quadados residuais } \\
\text { Error sum of square }\end{array}$ & $91.743,0725$ & $47.525,7031$ & $48.279,3708$ & $34.037,2898$ \\
\hline $\begin{array}{l}\text { Grau de liberade residual } \\
\text { Error degree of freedom }\end{array}$ & 7456 & 7055 & 7455 & 7054 \\
\hline $\mathrm{R}^{2}$ & 0,0178 & 0,4911 & 0,4699 & 0,6262 \\
\hline
\end{tabular}

${ }^{*} \mathrm{P}<0,0001 ;{ }^{1} \mathrm{GC}=$ grupo contemporâneo; ${ }^{2} \mathrm{e} . \mathrm{p} .=$ erro-padrão; $\mathrm{s} /=\mathrm{sem} ; \mathrm{c} /=\mathrm{com}$.

${ }^{*} P<.0001 ;{ }^{*} C G=$ contemporary group; ${ }^{2}$ s.e.= standard error. 
proporcionou maior estimativa para $\beta_{1}$, ocorrendo grande redução na soma de quadrados do resíduo. Além disso, este modelo estatístico se adequou melhor ao comportamento da variável $\left(\mathrm{R}^{2}=0,4911\right)$. Já no modelo de análise do PE contendo GC e peso do animal, este padrão não foi verificado. Constatou-se pequena variação nos valores estimados para os coeficientes de regressões, de acordo com o modelo contendo ou não o GC, $\beta_{1}=0,0695 \mathrm{e}$ $\beta_{1}=0,0708$, respectivamente, e $\beta_{2}=-0,0000415 \mathrm{e}$ $\beta_{2}=-0,0000413$, respectivamente (Tabela 3). Os resultados evidenciaram que a inclusão do GC no modelo para estimar o coeficiente de regressão da idade do animal evitaria a super ou subestimação das informações pertencentes a animais de grupos com idades extremas. Por outro lado, na correção do $\mathrm{PE}_{i}$ pelo $\mathrm{P} 570$ ( 2 a etapa), as pequenas diferenças entre os coeficientes de regressão estimados pelos modelos com e sem o GC indicaram que, com a consideração do peso do animal, grande parte do efeito do GC já era removida. Assim, o uso do modelo que considera o GC evitaria possíveis confundimentos entre os efeitos considerados, pois grupos de animais mensurados com menor idade, geralmente, apresentam menores PC e PE e vice-versa. Dessa maneira, a estimação dos coeficientes de regressão foi feita levando-se em conta os efeitos de GC, resultando nas seguintes equações de predição:

$$
\begin{aligned}
\hat{P E i}= & 25,7133950+0,03833497(I-574,6157147) \\
\hat{P E i p}= & 25,5475688+0,070814749(P 570-309,9618068) \\
& +(-0,000041334)\left[(P 570)^{2}-2 P 570(309,9618068)\right. \\
& \left.+(309,9618068)^{2}\right]
\end{aligned}
$$

O coeficiente de regressão linear estimado para a idade $(0,03833497 \mathrm{~cm} /$ dia $)$, nesta pesquisa, foi menor que os estimados, em modelos semelhantes, por BRITO (1997), em uma população de Polled Hereford variando de 317 a 489 dias de idade $(0,1483944 \mathrm{~cm} / \mathrm{dia})$, e DAL-FARRA et. al. (1998), utilizando animais Nelore entre 450 e 700 dias de idade $(0,138088 \mathrm{~cm} /$ dia). A menor taxa de aceleração de crescimento do PE no presente trabalho estaria indicando menor influencia da idade do animal na população em estudo. Por outro lado, os coeficientes de regressão do PC sobre o PE, nos respectivos estudos citados, foram semelhantes aos observados neste trabalho, a saber, $0,06969517 \mathrm{~cm} / \mathrm{kg}$ e $-0,00006005 \mathrm{~cm} / \mathrm{kg}^{2}$, entre 189 e $437 \mathrm{~kg}$, segundo

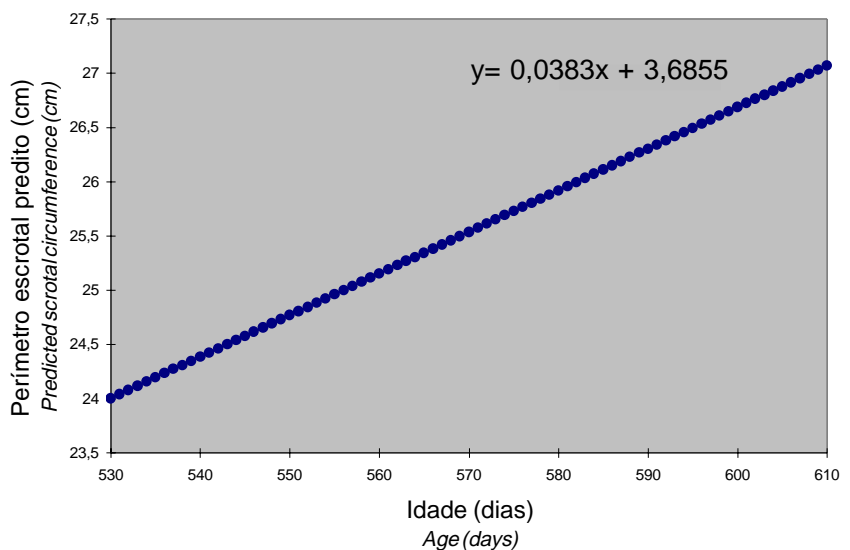

Figura 3 - Perímetro escrotal predito, de touros jovens Nelore, no Paraguai, em função da idade do animal.

Figure 3 - Predicted scrotal circumference (SC), of young Nellore bulls, in Paraguay, according to the animal age.

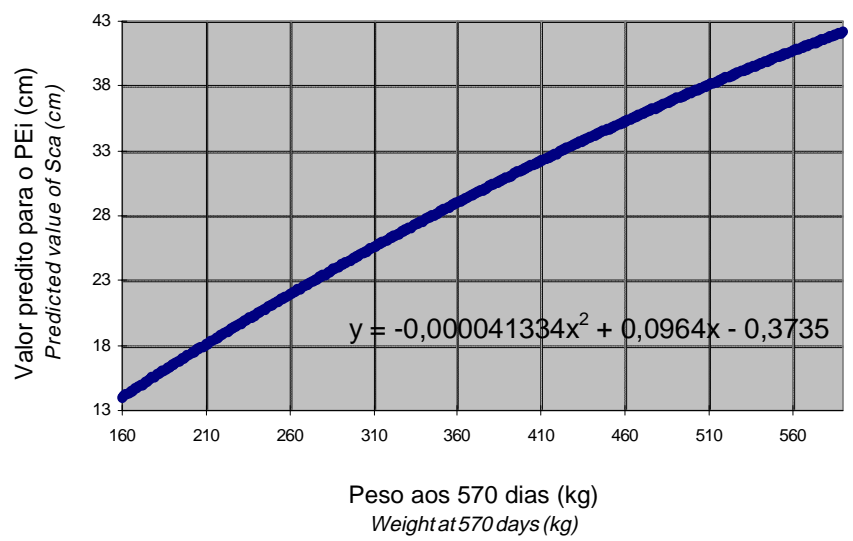

Figura 4 - Perímetro escrotal corrigido para 570 dias de idade (PEi) de touros jovens Nelore, no Paraguai, predito em função do peso corporal aos 570 dias.

Figure 4 - Corrected scrotal circumference for 570 days of age (SCa) of young Nellore bulls, in Paraguay, predicted according to the body weight at 570 days.

BRITO (1997), e 0,0794728 cm/kg e -0,00005778 cm/ $\mathrm{kg}^{2}$, entre 180 e $440 \mathrm{~kg}$, de acordo com DAL-FARRA et al. (1998), evidenciando que a influência do peso corporal em animais jovens parece ser mais consistente que a idade.

Nas Figuras 3 e 4, são apresentados os valores preditos do PE, em função da idade e do peso padronizado aos 570 dias, respectivamente. Os coeficientes de regressão estimados podem ser interpre- 
tados como funções do crescimento dos testículos dos animais. Assim, pode-se esperar que nesta faixa de idade, a cada 100 dias transcorridos, o PE cresça aproximadamente $3,83 \mathrm{~cm}$, em média, e, nas condições medidas, a cada $100 \mathrm{~kg}$ de peso ganho, o PE cresça, em média, ao redor de 7,08 cm.

A Figura 5 foi elaborada levando-se em conta os valores preditos do PE somente para a idade (linha anterior $-\hat{P E i}$ ) e a idade e o peso do animal (linha posterior - $P \hat{E} i p$ ), de acordo com a idade do animal. Analisando-se esta Figura, observa-se que o plano formado pela união da linha anterior $(\hat{P E i})$ com a posterior ( $P \hat{E}$ ip $)$ mostra o efeito da correção do PE pela idade e peso corporal em comparação com a correção apenas pela idade. Pelas equações utilizadas (1 e 2), o diferencial $\hat{P E i}$ - $P \hat{E} i p$ passa de negativo a positivo após a média de idade e peso considerados (570 dias e $310 \mathrm{~kg}$ de PC), notando-se, na Figura 5, que, a idades jovens, o PÊip é superior ao $\hat{P E} i$, e contrariamente, para animais mais velhos, a consideração do peso corporal determina $\mathrm{PE}$ menor do que aquele levando-se em conta apenas a idade. Assim, esta característica ajustada para idade e peso do animal poderia facilitar a diferenciação de animais menos precoces sexualmente, mas com elevados PE em idades adultas.

$\mathrm{Na}$ Tabela 4, encontra-se um extrato parcial dos fatores de correção estimados para o PE. Os valores iguais a 1 indicam idade ou peso para os quais o fator foi tomado como padrão, isto é, 570 dias de idade e $310 \mathrm{~kg}$ de P570. Observa-se que os fatores de correção estimados para idade variaram de 1,0638 para 530 dias a 0,9433 para 610 dias, apresentando amplitude de 0,12 , que foi menor que aquela dos

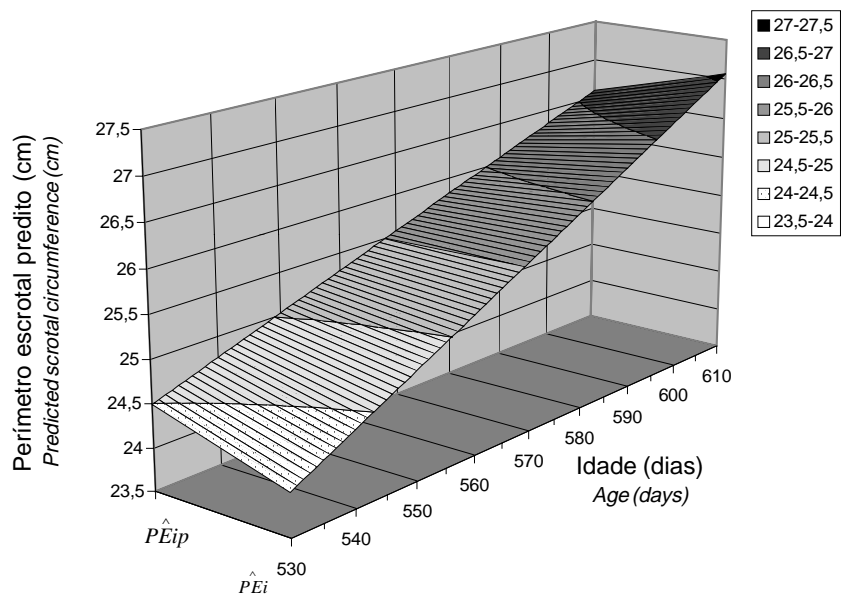

Figura 5 - Valores preditos do perímetro escrotal, em função da idade (linha anterior - PE $\hat{E}_{j}$ ) e idade e peso (linha posterior - $\left.\mathrm{PE} \hat{E}_{i p}\right)$, de touros jovens Nelore, no Paraguai.

Figure 5 - Predicted values of scrotal circumference (SC) according to the age (front line - $P \hat{E}_{i}$ ) and the age and body weight (back line - $P \hat{E}_{i p}$ ), of Nellore bulls, in Paraguay.

estimados para idade e peso do animal, que variou de 1,8252 para $160 \mathrm{~kg} \mathrm{a} 0,6063$ para $590 \mathrm{~kg}$ de P570, com amplitude de 1,22. DAL-FARRA et al. (1998) calcularam fatores de correção de $\mathrm{PE}$ de touros jovens Nelore, com amplitudes iguais a 0,46 para idade e 0,52 para PC. No presente estudo, a população apresentou-se mais heterogênea no peso corporal, havendo maior variação deste em menor intervalo de idade. Assim, nas condições deste estudo, a influência do peso do animal sobre o seu PE pareceu ser mais marcante do que a da sua idade.

Um exemplo de aplicação dos fatores de corre-

Tabela 4 - Fatores de correção para perímetro escrotal (PE), estimados para idade e $310 \mathrm{~kg}$ de peso aos 570 dias de idade do animal (P570), em touros jovens Nelore, no Paraguai

Table 4 - Correction factors for Scrotal circumference, estimated for age and $310 \mathrm{~kg}$ of weight at 570 days of age of the animal (W570), for young Nellore bulls, in Paraguay

\begin{tabular}{|c|c|c|c|}
\hline \multicolumn{2}{|c|}{$\begin{array}{l}1-\text { Etapa } \\
1^{\text {st }} \text { Step }\end{array}$} & \multicolumn{2}{|c|}{$\begin{array}{c}2^{\text {a }} \text { Etapa } \\
2^{\text {nd }} \text { Step }\end{array}$} \\
\hline $\begin{array}{c}\text { Idade (dias) } \\
\text { Age (days) }\end{array}$ & $\begin{array}{c}\text { Fator de correção } \\
\text { Correctionfactor }\end{array}$ & $\begin{array}{l}\mathrm{P} 570(\mathrm{~kg}) \\
W 570\end{array}$ & $\begin{array}{c}\text { Fator de correção } \\
\text { Correction factor }\end{array}$ \\
\hline 530 & 1,0638 & 160 & 1,8252 \\
\hline 540 & 1,0471 & 200 & 1,4802 \\
\hline 550 & 1,0309 & 240 & 1,2530 \\
\hline 560 & 1,0152 & 280 & 1,0924 \\
\hline 570 & 1,0000 & 310 & 1,0000 \\
\hline 580 & 0,9852 & 380 & 0,8431 \\
\hline 590 & 0,9708 & 450 & 0,7373 \\
\hline 600 & 0,9569 & 520 & 0,6619 \\
\hline 610 & 0,9433 & 590 & 0,6063 \\
\hline
\end{tabular}


ORTIZ PEÑA et al.

Tabela 5 - Exemplo da utilização dos fatores de correção do perímetro escrotal (PE) para uma pequena amostra de jovens touros da raça Nelore

Table 5 - Example of using the correction factors of scrotal circumference (SC) in a small sample of young Nellore bulls

\begin{tabular}{lccccc}
\hline Animal & $\begin{array}{c}\text { PE } \\
\text { observado }(\mathrm{cm}) \\
\text { Observed SC }\end{array}$ & $\begin{array}{c}\text { Idade } \\
(\text { dias }) \\
\text { Age (days) }\end{array}$ & $\begin{array}{c}\text { Peso vivo } \\
(\mathrm{kg}) \\
\text { Bodyveight }\end{array}$ & $\begin{array}{c}\text { PE corrigido para } \\
\text { idade }(\mathrm{cm}) \\
\text { SC corrected for age }\end{array}$ & $\begin{array}{c}\text { PE corrigido para } \\
\text { idade e peso (cm) } \\
\text { SC corrected for age } \\
\text { and body weight }\end{array}$ \\
\hline A & 38 & 560 & 450 & 38,58 & 28,44 \\
B & 38 & 560 & 380 & 38,58 & 32,53 \\
C & 27 & 530 & 280 & 28,72 & 31,38 \\
D & 26 & 570 & 310 & 26,00 & 26,00 \\
E & 24 & 530 & 240 & 25,53 & 31,99 \\
F & 21 & 540 & 150 & 21,99 & 40,13 \\
G & 35 & 610 & 380 & 33,01 & 27,83 \\
H & 15 & 540 & 200 & 15,71 & 23,25 \\
I & 18 & 550 & 240 & 18,56 & 23,25 \\
J & 40 & 660 & 520 & 37,73 & 24,98 \\
K & 28 & 530 & 380 & 29,79 & 22,11 \\
L & 28 & 630 & 380 & 26,41 & 27 \\
\hline
\end{tabular}

ção do perímetro escrotal pode ser encontrado na Tabela 5. Verifica-se que o criador poderia optar pela correção do $\mathrm{PE}$ somente para a idade do animal $\left(\mathrm{PE}_{i}\right)$ ou para idade e peso corporal $\left(\mathrm{PE}_{i p}\right)$. Observa-se que, para indivíduos com mesmo valor do perímetro escrotal e mesma idade, após a correção para idade e peso corporal, o animal de menor peso foi melhor classificado. Por outro lado, para animais com perímetros e pesos iguais e idades diferentes, a correção tanto para idade $\left(\mathrm{PE}_{i}\right)$ quanto para idade e peso $\left(\mathrm{PE}_{i p}\right)$ privilegiou o animal mais novo. A utilização do PE corrigido apenas para a idade poderia ser feita por criadores interessados em obter ganho genético para precocidade sexual e, também, em peso corporal, uma vez que deve haver resposta correlacionada para essa última característica. Por outro lado, criadores que procuram ganhos genéticos em precocidade sexual e/ou que não queiram aumentar o tamanho adulto de suas vacas de cria (por exemplo, o criador que comercializa bezerros desmamados) poderiam optar por corrigir o PE para idade e peso corporal. A correção para idade e peso corporal responderia a questão: se três animais têm $30 \mathrm{~cm}$ de PE e mesma idade, mas um pesa $200 \mathrm{~kg}$, outro $300 \mathrm{~kg}$ e o outro $400 \mathrm{~kg}$, qual deve ser o mais precoce sexualmente? Criadores de zebuínos, em geral, com tamanho de vacas já no ponto ideal, porém com deficiências de precocidade sexual, têm visto esta opção como vantajosa. Assim, a correção do perímetro para idade e peso corporal (PEip) auxiliaria na discriminação dos animais sexualmente mais precoces.
Estes resultados permitem concluir que os animais com maiores valores de PEip não necessariamente seriam os de maiores taxas de crescimento e/ou tamanho adulto. Assim, a utilização do PE corrigido para idade e peso do animal como critério de seleção para precocidade sexual, quando mensurado a idades mais jovens, poderia trazer progressos genéticos ao rebanho em precocidade sexual, sem, necessariamente, elevar os pesos/tamanhos adultos dos animais. No entanto, outros estudos envolvendo esta e outras características indicativas da conformação do peso corporal, em machos Nelore, seriam importantes para elucidar melhor as associações existentes entre os genes que determinam precocidade sexual e crescimento.

\section{Conclusões}

Os efeitos da idade do animal e do peso corporal foram importantes fontes de variação a serem removidas, quando considerado o perímetro escrotal como critério de seleção para melhorar, geneticamente, a precocidade sexual.

A inclusão do efeito de GC no modelo para estimar os fatores de correção do PE para a idade do animal foi eficiente para remover o efeito de idades extremas, que poderia ocasionar viés nos fatores de correção estimados.

O PE corrigido para idade e peso do animal ao sobreano mostrou ser um critério de seleção mais adequado que o PE corrigido apenas para idade. 


\section{Referências Bibliográficas}

ALBUQUERQUE, L.G., FRIES, L.A. Precocidad: estrategias de selección. In: SIMPOSIO LATINOAMERICANO PRODUCTIVIDAD EN GANADO DE CORTE, 1, 1998, Santa Cruz de la Sierra. Anais... São Paulo: DBO Sul, 1998. p.19-24.

ALENCAR, M.M., BARBOSA, P.F., BARBOSA, R.T. et al. 1993. Parâmetros genéticos para peso e circunferência escrotal em touros da raça Canchim. R. Soc. Bras. Zootec., 22(4):572583.

ALENCAR, M.M., VIEIRA, R.C. Crescimento testicular de touros da raça Canchim. Pesq. Agropec. Bras., 24(11):1329$1333,1989$.

ARIAS, A., SLOBODZIAN, A. Análisis de los datos de los registros de la raza Nelore de Paraguay. In: CONGRESO INTERNACIONAL DE PRODUCCIÓN DE CARNE, 1, 1998, Mariano R. Alonso. Anales... Asunción: APCN/ BURSAL/DBO Sul, 1998. p.51-94.

BARROS, A.J., ELER, J.P., FERRAZ, J B. et al. Estudo da influência de fatores não genéticos sobre a idade ao primeiro parto e dias para o parto de vacas da raça Nelore. In: REUNIÃO ANUAL DA SOCIEDADE BRASILEIRA DE ZOOTECNIA, 33, 1996, Fortaleza. Anais... Viçosa: SBZ, 1996. p.150-151.

BRINKS, J.S., McINERNEY, J.M., CHENOWETH, P.J. 1978. Relationships of age at puberty in heifers to reproductive traits in young bulls. Proc. West. Sec. Amerc. Soc. Anim. Sci., 29:28-30.

BRITO, F.V. Influência da idade e peso corporal sobre o perímetro escrotal em touros Hereford - estimativas de fatores de correção. In: REUNIÃO ANUAL DA SOCIEDADE BRASILEIRA DE ZOOTECNIA, 34, 1997, Juiz de Fora. Anais... Viçosa: SBZ, 1997. p.130-132.

COULTER, G.H., KELLER, D.G. 1982. Scrotal circumference of young beef bulls; relationship to paired testes weight effect to breed, and predictability. Can. J. Anim. Sci., 62:133-139.

DAL-FARRA, R.A., LOBATO, J.F., FRIES, L.A. 1998. Fatores de correção do perímetro escrotal para efeitos de idade e peso ao sobreano de tourinhos Nelore. R. Bras. Zootec., 27(6):1092-1096.

ELER, J.P., FERRAZ, J.B., SILVA, P.R. Estimação simultânea de parâmetros genéticos para características de importância econômica na raça Nelore, com a utilização de modelos animais. In: REUNIÃO ANUAL DA SOCIEDADE BRASILEIRA DE ZOOTECNIA, 33, Fortaleza, 1996. Anais... Viçosa: SBZ, 1996. p.99-101.

FRIES, L.A., ROSO, V.M. Conectabilidade em avaliações genéticas de gado de corte: uma proposta heurística. In: REUNÃO ANUAL DA SOCIEDADE BRASILEIRA DEZOOTECNIA, 34, Juiz de Fora, 1997. Anais... Viçosa: SBZ, 1997. p.159-161.
GRESSLER, S.L., BERGMANN, J.A., PENNA, V.M. et al Estudo das associações genéticas entre perímetro escrotal e características reprodutivas de fêmeas da raça Nelore. In: REUNÃO ANUAL DA SOCIEDADE BRASILEIRA DE ZOOTECNIA, 35, Botucatu, 1998. Anais... Viçosa: SBZ, 1998. p.368-370

LÔBO, R.B., DE LOS REYES, A., MAGNABOSCO, C. et al. Variância e covariância genética para circunferência escrotal e pesos em rebanhos Nelore. In: REUNIÃO ANUAL DA SOCIEDADE BRASILEIRA DE ZOOTECNIA, 31, 1994, Maringá. Anais...Viçosa: SBZ, 1994. p.163.

MARTINS FILHO, R., LÔBO, R.B., DE LOS REYES A. et al. Estimativas de parâmetros genéticos para a circunferência escrotal em bovinos da raça Nelore. In: REUNIÃO ANUAL DA SOCIEDADE BRASILEIRA DE ZOOTECNIA, 31, Maringá, 1994. Anais...Viçosa: SBZ, 1994. p.162.

PINEDA, N.R., FONSECA, V.O., PROENÇA, R.V. Comportamiento sexual del toro. In: CONGRESSO INTERNACIONAL DE PRODUCCIÓN DE CARNE, 1, 1998, Mariano R. Alonso. Anales... Asunción: APCN/BURSAL/ DBO Sul. 1998. p.17-22.

QUIRINO, C.R., BERGMANN, J. A. Herdabilidade do perímetro escrotal ajustado e não ajustado para peso corporal usando modelo animal uni e bivariado. In: REUNIÃO ANUAL DA SOCIEDADE BRASILEIRA DE ZOOTECNIA, 341997 , Juiz de Fora. Anais... Viçosa: SBZ, 1997. p.127-129.

SAS Institute Inc., SAS/STAT. User's Guide, version 6.11, 4.ed, v.2, Cary: SAS Institute Inc., 1996. 842p.

SILVA, A.E., DODE, M.A., UNANIAN, M.M. 1993 Avaliação da capacidade reprodutiva do touro. In: EMBRAPA. Capacidade reprodutiva do touro de corte: funções, anormalidades e fatores que a influenciam. Campo Grande, MS: EMBRAPA-CNPGC. p.128-11.

TOELLE, V.D., ROBISON, O.W. 1985. Estimates of genetic correlation between measurements and female reproductive traits in cattle. J. Anim. Sci., 60:89-100.

UNANIAM, M.M. A procura de marcadores de precocidade em gado Nelore. In: O NELORE DO SÉCULO XXI. NELORE PRECOCE: SELEÇÃO, PRODUÇÃOECOMERCIALIZAÇÃO, 4, 1997, Uberaba. Anais...Uberaba: ABCZ-ABCN, 1997. p.51-57.

Recebido em: 12/05/99

Aceito em: 07/06/00 